\title{
(1⿶)
}

Citation:

Kime, NH (2013) Weight loss in type 2 diabetes. Independent Nurse. 2 - 6 (5). ISSN 1747-9800

Link to Leeds Beckett Repository record:

https://eprints.leedsbeckett.ac.uk/id/eprint/113/

Document Version:

Article (Accepted Version)

The aim of the Leeds Beckett Repository is to provide open access to our research, as required by funder policies and permitted by publishers and copyright law.

The Leeds Beckett repository holds a wide range of publications, each of which has been checked for copyright and the relevant embargo period has been applied by the Research Services team.

We operate on a standard take-down policy. If you are the author or publisher of an output and you would like it removed from the repository, please contact us and we will investigate on a case-by-case basis.

Each thesis in the repository has been cleared where necessary by the author for third party copyright. If you would like a thesis to be removed from the repository or believe there is an issue with copyright, please contact us on openaccess@leedsbeckett.ac.uk and we will investigate on a case-by-case basis. 


\section{Weight loss in people with type 2 diabetes}

\section{Prevalence of type 2 diabetes}

Currently, the estimated global prevalence of diabetes is over 285 million and this figure is expected to increase to 438 million people by 2030. In the UK, since 1996, the incidence of people diagnosed with diabetes has increased from 1.4 million to 2.6 million. This number continues to rise and most health experts agree that by 2025 over 4 million people will have diabetes. The majority of cases will be type 2 diabetes because of our ageing population and the rising numbers of people, including children and adolescents, who are overweight and obese. ${ }^{1}$ For this reason, diabetes represents one of the biggest health challenges affecting the UK.

\section{Type 2 diabetes and obesity}

Type 2 diabetes has a very strong association with overweight and obesity. ${ }^{2}$ Between 80 and $90 \%$ of people with type 2 diabetes are overweight and approximately $60-90 \%$ of type 2 diabetes is obesity related. ${ }^{3} \mathrm{As} \mathrm{BMI}$ increases so does the risk of developing type 2 diabetes. In fact, the prevalence of type 2 diabetes is 3-7 times higher in obese than in normal weight adults and those with a BMI of more than $35 \mathrm{~kg} / \mathrm{m}^{2}$ are 20 times more likely to develop diabetes than those with a BMI of between 18.5 and $24.9 \mathrm{~kg} / \mathrm{m}^{2}{ }^{4}$. The link between obesity and type 2 diabetes is an important one in terms of health outcomes, not least because obesity is an independent risk factor for cardiovascular disease, which is the major cause of death in those with type 2 diabetes. This is compounded by the fact that obesity complicates the management of type 2 diabetes as it increases insulin resistance and blood glucose concentrations. Therefore, it makes sense to maintain a lower body weight in order to reduce long-term morbidity and mortality from type 2 diabetes and its related complications. Even a moderate weight-loss has been shown to reduce the need for diabetes medications, improve glycaemic control and reduce the risk of cardiovascular disease and other co-morbidities associated with type 2 diabetes. ${ }^{5,6}$

\section{Weight management}

For this reason, increasingly, the need to integrate weight management into diabetes care is being recognised. Diabetes UK advocates that weight-loss in people with type 2 diabetes who are overweight or obese should be the primary management approach. This involves developing strategies for achieving and maintaining a healthy body weight through lifestyle modifications that address energy intake and energy expenditure, i.e. diet and physical activity.

\section{Diet}

The main requirement of a dietary approach to weight-loss is that total energy intake should be less than energy expenditure. ${ }^{7}$ Whilst there is no universally agreed target for achieving this, most experts recommend decreasing energy intake by between 500 and $1000 \mathrm{kcal}$ per day. ${ }^{4}$ A variety of dietary strategies are used to help individuals lose weight, including lowfat diets, low-carbohydrate diets and meal replacements. In relation to type 2 diabetes, a low fat, high carbohydrate diet in combination with regular exercise is the traditional recommendation for treating the condition. Although, evidence from various randomised controlled trials in recent years has shown that low carbohydrate diets are comparable or better than traditional low fat, high carbohydrate diets for weight reduction. However, the 
role of low carbohydrate diets for weight loss and improving glycaemic control is controversial because of their association with the popular Atkins diet. Although some form of low carbohydrate diet may be a viable option for certain individuals, the key factor with this and all dietary strategies is that, whilst many of these produce short-term benefits, their main limitation is poor long-term compliance and weight regain. Furthermore, it is impossible to identify the most effective dietary strategy since it is unlikely that any single diet will be the optimal diet for all those with type 2 diabetes who are overweight or obese. Encouraging people to adopt a diet that suits them is more likely to improve outcomes since it is the degree of adherence rather than the type of strategy that seems to be the most effective in achieving weight-loss. ${ }^{8}$ Therefore, it makes sense to tailor dietary advice to the individual. A successful nutrition strategy is patient-centred and takes into account dietary preferences. It is also one that is capable of being adapted to suit changing lifestyles and can be followed for the long-term, both during the weight-loss and weight maintenance phases. $^{9}$

\section{Physical activity}

In order to lose weight an individual needs to use more calories than they consume. Therefore, physical activity is an essential component of any weight management programme. Well-documented evidence demonstrates the importance of physical activity for everyone, especially those who are overweight or obese and have co-morbidities, ${ }^{10}$ although physical activity as a stand-alone strategy it is not effective for weight loss in people with type 2 diabetes. However, a combination of physical activity and diet is beneficial since this combination results in greater weight loss initially, helps to maintain weight loss and prevents weight gain. Regular exercise also improves insulin sensitivity and glycaemic control, has a positive effect on cardiovascular risk and may reduce the overall mortality in people who have type 2 diabetes. As with dietary strategies, it is the degree of adherence to an activity programme that will predict outcomes rather than the type of activity. A structured programme that involves planned and repetitive exercise may not work for some people, whereas encouraging an individual to move more regularly as part of their daily routine, i.e. walking or using the stairs, may be more effective in ensuring compliance. Therefore, it is important to tailor any activity to an individual's lifestyle and introduce it gradually, increasing the amount of activity as fitness improves.

\section{Weight maintenance}

Long-term maintenance of weight loss rather than initial weight loss is the biggest challenge for most people, including those with type 2 diabetes. Those who follow a weight loss programme can lose $5-10 \%$ of their body weight in approximately 6 months, but for longterm benefits weight maintenance strategies are necessary. Some strategies that are associated with successful long-term weight loss include: eating a diet low in calories, fats and sugars; smaller portion sizes; fewer snacks and meals away from home; monitoring nutritional intake and body weight; consistent eating habits incorporating breakfast on a daily basis; participation in regular physical activity and effective support mechanisms. In addition, weight loss attainment, however small, on a gradual and regular basis is a successful predictor of long-term weight maintenance. 


\section{Structured education}

NICE guidelines for the management of type 2 diabetes indicate that structured education should be an integral part of diabetes care and needs to include dietary and physical activity components. Although it is recognised that diabetes education should be tailored to meet the needs of the individual, there is a lack of evidence relating to the type of interventions that are the most effective in bringing about weight loss. This makes it difficult to determine which aspects are most important for facilitating behaviour change and should be incorporated into practice. It is clear that for any approach to result in long-lasting behaviour change it needs to be recognised that people with diabetes are in control of their lives on a day-to-day basis and make their own choices about their lifestyles, including dietary intake and physical activity. Therefore, a person-centred approach is more likely to be successful than one where the health care professional (HCP) is in control and makes all the decisions. In addition, the evidence suggests that if HCPs support individuals' autonomy they will be more motivated to self-care and this ultimately results in improved glycaemic control.

\section{The role of nurses in weight management}

Achieving and sustaining long-term changes to dietary and physical activity behaviours is extremely difficult for the majority of people and perhaps more so for those with type 2 diabetes. Therefore, the support and encouragement that individuals with type 2 diabetes receive from their HCPs is vitally important. However, HCPs are in a difficult position since people can access a huge amount of information about weight loss through the media, some of which may conflict with recommended advice. This is compounded by the fact that there seems to be little consensus as to what actually works. This aside, tackling such a serious public health concern as increasing overweight and obesity amongst those with type 2 diabetes necessarily has to involve HCPs at all levels and in all settings. All members of the multi-disciplinary team (MDT) must be able to deliver and implement dietary and physical activity advice in an appropriate way. In order to do this, the necessary resources need to be in place to train HCPs and ensure they are allowed sufficient time to develop and maintain their skills. Fundamentally, achieving weight loss has to be part of a team approach that allows individuals with type 2 diabetes to be at the centre of the decision-making process.

The role that nurses play in weight management is crucial because of their unique skills and their position within the MDT. Although a registered dietitian with specialist knowledge frequently takes the lead in nutritional care, in reality it is more likely to be the district or practice nurse who is able to provide the continuity of care and on-going support that individuals benefit from. All nurses in all settings will have contact with people with type 2 diabetes and are, therefore, ideally placed to provide weight management education and support to those with the condition. In addition, nurses are in a better position to give their time at key points when individuals most need help. Nurses are also well placed to work with other members of the MDT and other allied health professionals, for example, doctors, podiatrists, pharmacists and dietitians. They are not only able to improve the knowledge and skills of individuals with type 2 diabetes, but also their colleagues' through appropriate education programmes and teamwork. In addition, nurses are often the main link between individuals with type 2 diabetes and other agencies, for example walking support groups; groups such as these are often a key contributory factor towards an individual achieving and maintaining weight loss. 
Compassion, understanding and sensitivity are key attributes that nurses can utilise to help people with type 2 diabetes, especially when individuals have been unable to lose weight or are struggling to achieve their goals. Individuals need to feel that they are supported and should not be made to feel guilty about their lifestyles or that they are to blame. Several techniques can be used to assist those with type 2 diabetes in losing weight. Initially, problem or difficult areas and behaviours can be identified and specific and realistic goals agreed. Research has demonstrated that small and achievable targets allow individuals to experience success more readily, which in turn motivates them to continue with their weight loss programme. Strategies such as self-monitoring (daily records of food intake and physical activity), stimulus control (avoiding triggers that prompt eating), reinforcement (revisiting agreed goals) and problem-solving (identifying barriers and ways to overcome them) can then be used to facilitate behavioural change. ${ }^{4}$ In addition, frequent contact with a HCP needs to be scheduled into any weight management programme since this is associated with better long-term weight loss maintenance.

\section{Conclusion}

The figures for type 2 diabetes are alarming and confirm that this condition represents the biggest health challenge facing the UK today. In order to see a reduction in the number of people dying from type 2 diabetes and its associated co-morbidities, there needs to be an increased awareness of the risks, large-scale changes in lifestyle and improved selfmanagement among people with type 2 diabetes, in particular those who are overweight or obese. A collaborative approach is required to address the risks of an unhealthy diet and sedentary lifestyle, and to help people with type 2 diabetes achieve effective selfmanagement. Health care professionals, especially nurses, have their part to play, by working closely with individuals to set tailored strategies and realistic expectations that can help them work towards their diabetes management and weight loss goals.

\section{Box 1}

Recommendations ${ }^{11}$

- Weight management should be the primary nutritional strategy in managing glucose control in type 2 diabetes for people who are overweight or obese.

- Weight reduction for the overweight or obese person with type 2 diabetes has been shown to be effective in improving glycaemic control, cardiovascular risk factors and other co-morbidities.

- The main requirement of a dietary approach to weight loss is that total energy intake should be less than energy expenditure.

- The focus should be on total energy intake rather than the source of energy in the diet (macronutrient composition) for optimal glycaemic control.

- The total amount of carbohydrate consumed is a strong predictor of glycaemic response and monitoring total carbohydrate intake remains a key strategy in achieving glycaemic control.

- Low glycaemic index diets may reduce $\mathrm{HbA}_{1 \mathrm{c}}$ by up to $0.5 \%$.

- Regular, moderate physical activity can reduce $\mathrm{HbA}_{1 \mathrm{c}}$ by $0.45-0.65 \%$ independent of weight loss. 


\section{Box 2}

Across Great Britain, only $39 \%$ of men and $29 \%$ of women are meeting recommended physical activity levels. The Department of Health recommends that everyone, including those with type 2 diabetes, should strive to achieve at least 30 minutes of moderate intensity physical activity a day, on five or more days a week. This amount of activity may seem achievable for most people, but for those who are overweight or obese and lead a sedentary lifestyle, even this modest amount may seem overwhelming. Therefore, it is important to tailor physical activity to the individual introducing it gradually and increasing the amount as people improve their fitness. Physical activity does not need to occur in a single session to be beneficial, but can be divided into multiple, short bouts. Nor does it need to be part of a structured programme that has cost and time implications, for example, using a gym. Encouraging individuals to move more as part of their daily routine, for example walking or using the stairs, can produce similar benefits and also enhance compliance in the long-term.

\section{Box 3}

Achieving weight loss for those with type 2 diabetes needs to be part of a team approach that allows individuals to be at the centre of the decision-making process. All nurses in all settings will have contact with people with type 2 diabetes and are ideally placed to offer weight management education, support and encouragement to those with the condition. The role that nurses play within the team is crucial because of their unique skills. Compassion, understanding and sensitivity are key attributes, especially when individuals have been unable to lose weight or are struggling to achieve their goals. Individuals need to feel that they are supported and should not be made to feel guilty about their lifestyles or that they are to blame. Several techniques can be used to assist those with type 2 diabetes in losing weight. Initially, problem or difficult areas and behaviours can be identified and specific and realistic goals agreed. Strategies such as self-monitoring, stimulus control, reinforcement and problem-solving can then be used to facilitate behavioural change.

\section{References}

1. Diabetes UK. Diabetes in the UK 2012 - key statistics on diabetes. London: Diabetes UK; 2012.

2. Craig R, Mindell J (eds.). Health Survey for England 2011. London: The Information Centre; 2012.

3. Anderson JW, Kendall CWC, Jenkins DJA. Importance of weight management in type 2 diabetes: review with meta-analysis of clinical studies. J Am Coll Nutr. 2003;22(5):331-339.

4. Klein S, Sheard NF, Pi-Sunyer X, Daly A, Wylie-Rosett, J, Kulkarni K, et al. Weight management through lifestyle modification for the prevention and management of type 2 diabetes: rationale and strategies. Diabetes Care. 2004;27(8):2067-2073.

5. American Diabetes Association [ADA]. Nutrition recommendations and interventions for diabetes. Diabetes Care. 2006;29:2140-2196. 
6. American Diabetes Association [ADA]. Standards of medical care in diabetes. Diabetes Care. 2009;32(Suppl 1):S13-S61.

7. Dyson PA, Kelly T, Deakin T, Duncan A, Frost G, Harrison Z. Diabetes UK evidence-based nutrition guidelines for the prevention and management of diabetes. Diabet Med. 2011;28:1282-1288.

8. Drummond S. Obesity: a diet that is acceptable is more likely to succeed. I Fam Health Care. 2007;17:219-221.

9. Davis N, Forbes B, Wylie-Rosett J. Nutritional strategies in type 2 diabetes. Mt Sinai J Med. 2009;76:257-268.

10. NICE. Obesity: the prevention, identification, assessment and management of overweight and obesity in adults and children. London: NICE; 2006.

11. Diabetes UK. Evidence-based nutrition guidelines for the prevention and management of diabetes. London: Diabetes UK; 2011. 